\title{
LIQUITOPY®: A Liquid Tunable Microscope to Study Chromatin Organization in the Cell Nucleus.
}

Alberto Diaspro ${ }^{1,2,3}$, Nicholas Anthony ${ }^{1}$, Paolo Bianchini ${ }^{1,3}$, Isotta Cainero ${ }^{1,2}$, Melody Di Bona ${ }^{1,2}$, Luca Lanzanò $^{1,2}$, Aymeric Le Gratiet ${ }^{1}$, Riccardo Marongiu ${ }^{1,2}$, Michele Oneto ${ }^{1}$, Simone Pelicci ${ }^{1,2}$, Luca Pesce $^{1,2}$

1. Nanoscopy, Istituto Italiano di Tecnologia, Genova, Italy.

2. Department of Physics, University of Genoa, Genova, Italy.

3. Nikon Imaging Center, Istituto Italiano di Tecnologia, Genova, Italy.

The possibility of integrating different light-matter interactions to form images in optical microscopy is the starting point for the design and realization of LIQUITOPY ${ }^{\circledR}$, a "liquid tunable microscope". This could represent a new paradigm in data collection and image formation with a potential high impact in biophysics. Liquid takes inspiration from the philosophical and sociological speculation by Zygmunt Bauman [1] and tunable from the fact that, today, we have at our disposal methods allowing to "tune" the microscope across a large, almost unlimited, range of spatial and temporal resolution [2]. It is liquid because it aims to overlap in an efficient and optimized way different mechanisms of contrast and it is tunable because it offers a real time tunability regarding spatial and temporal resolution like a radio tuned on the preferred radio station. It is smart because can adapt the current configuration to the scientific question and is open to additional light-matter interaction modules. In our lab, we have implemented both classical and original solutions capable of managing most of the aspects of modern fluorescence microscopy. Important results have been obtained in single molecule localization methods [3], using STED-like configurations[4] and label-free approaches [5].

Light sheet microscopy, image scanning microscopy and expansion microscopy have been developed with the aim of deciphering key questions in the life sciences, including correlation spectroscopy [6]. The perspective related to label free approaches is growing since at the nanoscale dimension of labels counts. Three different main directions will be explored within LIQUITOPY ${ }^{\circledR}$ framework, namely: i) intrinsic fluorescence of biological macromolecules within the super-resolved fluorescence microscopy scenario considering interchangeable encoding and decoding of space and time at the nanoscale from lifetime to correlation spectroscopy from pump-probe to single molecule behaviour; ii) label-free unlimited super-resolved microscopy in transmission exploiting the saturation of the absorption states of the molecules under investigation within a pump-probe temporal window, non-linear interactions including multi photon excitation and high-order harmonic generation; iii) label-free microscopy based on Mueller and Jones matrix signatures coming from angular scattering processes and exploiting differential polarisation interactions and refractive index mismatches in the VIS-IR regions [7]. Aspects related to the different signal-to-noise ratio and to variable spatial/temporal resolutions can be technically solved and mathematically analyzed. What is relevant it is that they are bringing unique multimodal capabilities when collected all together.

LIQUITOPY® is designed with the aim of bringing a contribute to answer a key question in cellular and molecular biology: what are the local and global 4D (x,y,z,t) chromatin structures in the nucleus that rule the compaction and function of the human genome in the interphase cells and mitotic chromosomes? 
We aim to achieve: 1) the painting, at single cell level, of a 4D quantitative vision of those organizational motifs that today are not completely demonstrated about chromatin organization; 2) an optimal strategy for correlating images produced by different approaches from multiphoton to confocal microscopy, from expansion to light sheet microscopy and from super-resolved fluorescence to labelfree approaches. For example, the S(1,4) element of the Mueller matrix, named CIDS (Circular Intensity Differential Scattering), will provide a label-free scouting of nuclear regions with different chromatinDNA organizational motifs [8]. Our interest is related to imaging the physiological chromatin organization and potentially pathological changes induced by DNA damage, DNA damage response and repair focusing on: i) the possible interference of transcription and replication processes during oncogene-induced replicative stress, ii) the expression and activity of PARP1 in prostate cancer and iii) the Hutchinson-Gilford progeria syndrome linked mutations in the LMNA.

References:

[1] Z.Bauman, Liquid Modernity, Polity Press, Cambridge, 2000

[2] S.W.Hell et al, J. Phys. D: Appl. Phys. 48(44) (2015), p. 443001.

[3] F.Cella Zanacchi et al, Nature Methods 8(12) (2011), p. 1047.

[4] G.Vicidomini, P.Bianchini, A.Diaspro Nature methods (2018) online

[5] P.Bianchini and A.Diaspro, J Biophotonics 1(6) (2011), p. 443.

[6] L Scipioni et al. Nature Communications Biology 1(1) (2018), p. 10.

[7] N.Mazumder et al, Journal of Optics 19(2) (2017), p. 025301.

[8] S.Cully et al, Nature Methods (2018) online.

[9] A.Diaspro et al, IEEE Trans Biomed Eng 38(7) (1991), p. 670.

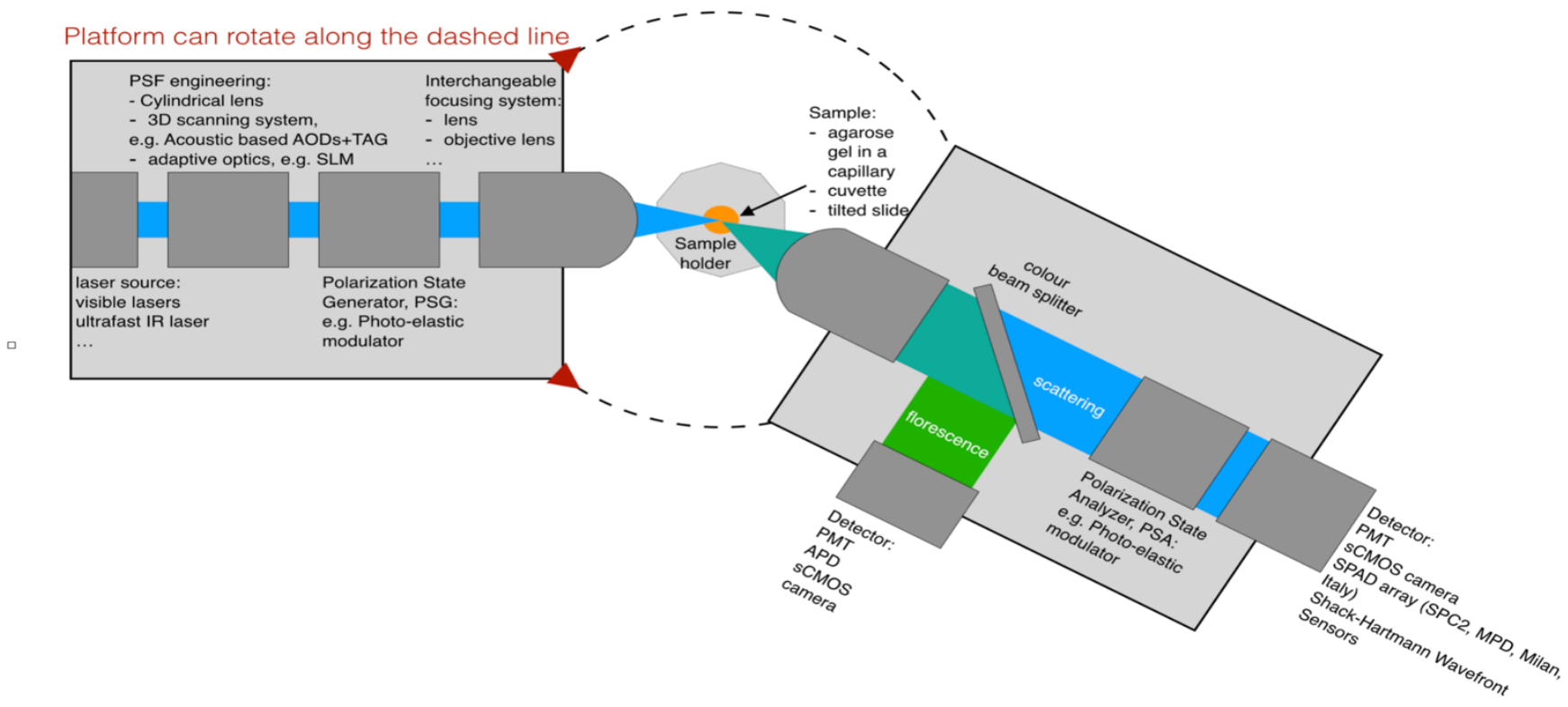

Figure 1. LIQUITOPY ${ }^{\circledR}$ scheme. 